\title{
Sinusitis, CTCAE
}

National Cancer Institute

\section{Source}

National Cancer Institute. Sinusitis, CT CAE. NCI Thesaurus. Code C143832.

A disorder characterized by an infectious process involving the mucous membranes of the paranasal sinuses. 